\title{
Effect of NaCl Salt on Vegetative Growth and Yield of Sixteen Tomato Lines
}

\author{
I. H. Shiam ${ }^{1}$, A. S. M. Nahiyan ${ }^{2}$, K. Momena ${ }^{2}$, H. Mehraj ${ }^{1}$ and A. F. M. Jamal Uddin ${ }^{1 *}$ \\ Dept. of Horticulture, Sher-e-Bangla Agricultural University, Dhaka ${ }^{1}$ \\ Advanced Seed Research and Biotech Center, ACI Limited, Dhaka, Bangladesh ${ }^{2}$ \\ Corresponding author*: jamal4@yahoo.com
}

\begin{abstract}
An experiment was conducted at the Sher-e-Bangla Agricultural University, Bangladesh to evaluate influence of salt $(\mathrm{NaCl})$ on sixteen tomato lines. Sixteen lines coded from $V_{1}$ (Line-O1) to $V_{16}$ (Line-16) were executed under different $\mathrm{NaCl}$ salinity conditions $\left(S_{0}:\right.$ Control; $S_{1}: 12 \mathrm{dS} / \mathrm{m}$ and $S_{2}: 16 \mathrm{dS} / \mathrm{m}$ ) following completely randomized design with three replications. Apart from control, $V_{8}$ provided tallest plant in $12 \mathrm{dS} / \mathrm{m}(43.7 \mathrm{~cm})$ and in $16 \mathrm{dS} / \mathrm{m}(38.4 \mathrm{~cm})$ salinity level at 60 days after transplanting which was statistically similar with the $V_{9}$ tomato line. $V_{8}$ line provided the maximum number of leaves per plant except control $(24.2$ and $21.1 \mathrm{in} 12 \mathrm{dS} / \mathrm{m}$ and $16 \mathrm{dS} / \mathrm{m}$ respectively). $V_{9}$ line produced maximum leaf area $\left(123.7 \mathrm{~cm}^{2}\right.$ and $97.6 \mathrm{~cm}^{2}$ in $12 \mathrm{dS} / \mathrm{m}$ and $16 \mathrm{dS} / \mathrm{m}$ respectively) under saline conditions which was followed by $V_{8}$ line $\left(112.7 \mathrm{~cm}^{2}\right.$ and $92.6 \mathrm{~cm}^{2}$ in $12 \mathrm{dS} / \mathrm{m}$ and $16 \mathrm{dS} / \mathrm{m}$ respectively). Maximum number of bunch per plant was observed from $V_{9}$ line (10.7 and 9.3 in $12 \mathrm{dS} / \mathrm{m}$ and $16 \mathrm{dS} / \mathrm{m}$ respectively) followed by $V_{8}$ line $(9.3$ and 9.3 in $12 \mathrm{dS} / \mathrm{m}$ and $16 \mathrm{dS} / \mathrm{m}$ respectively) except control. Maximum yield was found from $V_{9}$ line $(0.923 \mathrm{~kg} / \mathrm{plant})$ followed by $V_{2}$ line $(0.493 \mathrm{~kg} / \mathrm{plant})$ in $12 \mathrm{dS} / \mathrm{m}$ salinity level and maximum yield was found from $V_{9}$ line $(0.593 \mathrm{~kg} / \mathrm{plant})$ which was closely followed by $V_{8}$ line $(0.407 \mathrm{~kg} / \mathrm{plant})$ in $16 \mathrm{dS} / \mathrm{m}$ salinity level. Tomato line-09 was found the best tomato cultivar for salt affected areas in Bangladesh.
\end{abstract}

Key words: Tomato lines, salinity, growth and yield attributes

\section{Introduction}

Tomato (Solanum lycopersicum L.) belongs to the Solanaceae family is one of the important vegetable in Bangladesh and total production is low as compared to total demand. Soil salinity causes significant reductions of crop growth and development (Ashraf and Wahed, 1993) also yield (Tavakkoli et al., 2011; Hajiboland et al., 2009). Large amounts of land in southern region of Bangladesh remain uncultivable due to high level of soil salinity. The affected areas of Bangladesh are increasing rapidly (SRDI, 2010). Two recent devastating cyclonic disaster 'Aila in 2009' and 'Sidr in 2007' and excavated flood control embankment has increased the level of salinity. Due to increasing salinity affected areas normal agricultural land use practices become more restricted thus production levels decreased (Rahman and Ahsan, 2001). To overcome the salinity problem saline soils can be used to grow salt-tolerant plants (Ashraf and McNeilly, 2004). Thus development of salt tolerant crops is a key global agricultural goal (Ghanem et al., 2011). Tomato plant is moderately tolerant to salinity stress (Foolad, 2004; Peralta et al., 2005) and can tolerate salinity up to $2.5-2.9 \mathrm{dS} / \mathrm{m}$ in the root zone without yield losses (Sonneveld and Van der Burg, 1991) but exact salinity level may depending on cultivar sensitivity (Caro et al., 1991; Allen et al., 1998) and environmental conditions (Karlberg et al., 2006). Screening can be an easier 
method to determine salt tolerant genotypes. Considering above points, present study was to evaluate the ability of tomato lines under different saline conditions and screen out for salinity tolerant lines.

\section{Materials and Methods}

Experimental site and period: The experiment was conducted at Horticultural Farm, Sher-e-Bangla Agricultural University, Bangladesh during the months of November 2012 to March 2013.

Design and treatments of experiment: Sixteen tomato lines coded from $\mathrm{V}_{1}$ (Line-1) to $\mathrm{V}_{16}$ (Line-16) were executed under different salinity levels $\left(S_{0}\right.$ : Control; $S_{1}: 12 \mathrm{dS} / \mathrm{m}$ and $\left.S_{2}: 16 \mathrm{dS} / \mathrm{m}\right)$ using completely randomized design with three replication. In this study, $12 \mathrm{dS} / \mathrm{m}$ and $16 \mathrm{dS} / \mathrm{m}$ salinity levels were used to screen out the suitable variety for the highly saline affected areas like S3 (salinity level ranged from 8.1 $12.0 \mathrm{dS} / \mathrm{m}$ ) and S4 (12.1-16.0 dS/m) regions in Bangladesh (SRDI, 2010).

Genetic materials: Tomato lines were collected from local home and abroad. Seeds were sown on the regular seedbed in $5^{\text {th }}$ October and seedlings were transplanted into pots on $4^{\text {th }}$ November.

Fertilizers: Firstly 4 square meter plot was prepared with 4 inches tilling depth and entire soil of that plot was weighted. Then Cowdung (10 t/ha), Urea (300 kg/ha), Triple Super Phosphate (200 kg/ha), Muriate of Potash (220 kg/ha) (Mondal et al., 2011) for $8 \mathrm{~kg}$ soil was calculated. The entire amount of organic manure, Triple Super Phosphate and half of the Muriate of Potash were applied during final soil preparation. The remaining half of MP and entire urea applied in three equal installments, $1^{\text {st }}$ at 15 days after planting, $2^{\text {nd }}$ at flowering and the $3^{\text {rd }}$ at fruit maturity stages.

Application of treatments: The pot was filled with $8 \mathrm{~kg}$ soil having no salinity. Edible salt manufactured by Advanced Chemical Industries (ACI) Limited was used to create saline water. For the control plants were not exposed to salinity; whereas for the $12 \mathrm{dS} / \mathrm{m}$ and $16 \mathrm{dS} / \mathrm{m}$ salinity level $6.6 \mathrm{~g}$ ACI salt/L of water and $8.8 \mathrm{~g} \mathrm{ACI}$ salt $/ \mathrm{L}$ of water (the conversion rate was $1 \mathrm{dS} / \mathrm{m}=0.55 \mathrm{~g} / \mathrm{L}$ ) were given to each pot respectively. Saline solutions were given in the plastic bowl which was placed under the pots.

Data collection: Data were collected on plant height, number of leaves, leaf area, chlorophyll content, number of bunch/plant, days to fruiting (visual observation), number of flower/plant, number of fruit/plant, single fruit weight and yield/plant. Electronic precision balance was used for measuring fruit weight and yield/plant respectively.

Statistical analysis: Collected data were statistically analyzed using MSTAT-C program and mean was calculated. Differences of means between treatments were evaluated by Least Significance Difference (LSD) test at 5\% level of significance (Gomez and Gomez, 1984).

\section{Plant height}

\section{Results}

Plant height of tomato cultivars varied significantly at different days after transplanting also at different salinity levels. It was found that $\mathrm{V}_{4}$ provided the tallest $(61.3 \mathrm{~cm})$ plant in control whereas $\mathrm{V}_{8}$ provided the tallest plant in $12 \mathrm{dS} / \mathrm{m}(43.7 \mathrm{~cm})$ and $16 \mathrm{dS} / \mathrm{m}(38.4 \mathrm{~cm})$ salinity level at 60 days after transplanting which was statistically similar with the $\mathrm{V}_{9}$ (Figure 1 ). 


\section{Number of leaves}

Leaves number of tomato cultivars varied significantly at different days after transplanting also at different salinity levels. It was found that $\mathrm{V}_{8}$ provided the maximum number of leaves/plant $(31.3,24.2$ and 21.1 in control, $12 \mathrm{dS} / \mathrm{m}$ and $16 \mathrm{dS} / \mathrm{m}$ respectively) at 60 days after transplanting which was statistically similar with the $\mathrm{V}_{9}$ (Figure 2).

\section{Leaf area}

Leaf area was measured at 60 days after transplanting and showed significant differences among the variety at different salinity levels. In control, maximum leaf was produced by $\mathrm{V}_{8}\left(180.8 \mathrm{~cm}^{2}\right)$ followed by $\mathrm{V}_{3}\left(157.7 \mathrm{~cm}^{2}\right)$ while minimum from $\mathrm{V}_{11}\left(97.9 \mathrm{~cm}^{2}\right)$ (Table 1). On the other hand, $\mathrm{V}_{9}$ provided maximum leaf area $\left(123.7 \mathrm{~cm}^{2}\right.$ and $97.6 \mathrm{~cm}^{2}$ in $12 \mathrm{dS} / \mathrm{m}$ and $16 \mathrm{dS} / \mathrm{m}$ respectively) under saline conditions which was followed by $\mathrm{V}_{8}\left(112.7 \mathrm{~cm}^{2}\right.$ and $92.6 \mathrm{~cm}^{2}$ in $12 \mathrm{dS} / \mathrm{m}$ and $16 \mathrm{dS} / \mathrm{m}$ respectively) (Table 1$)$.

\section{Chlorophyll content}

Chlorophyll content was also measured at 60 days after transplanting and showed significant differences among the variety at different salinity levels. However maximum chlorophyll content was observed in $\mathrm{V}_{4}$ $\left(60.8 \%, 51.5 \%\right.$ and $47.4 \%$ in control, $12 \mathrm{dS} / \mathrm{m}$ and $16 \mathrm{dS} / \mathrm{m}$ respectively) while minimum in $\mathrm{V}_{9}(39.7 \%$, $30.6 \%$ and $25.6 \%$ in control, $12 \mathrm{dS} / \mathrm{m}$ and $16 \mathrm{dS} / \mathrm{m}$ salinity level respectively) which was statistically similar with $\mathrm{V}_{8}(40.7 \%, 31.3 \%$ and $25.8 \%$ in control, $12 \mathrm{dS} / \mathrm{m}$ and $16 \mathrm{dS} / \mathrm{m}$ salinity level respectively) (Table 1).

Table 1. Leaf area and chlorophyll content of tomato lines at different salinity level ${ }^{\mathrm{x}}$

\begin{tabular}{|c|c|c|c|c|c|c|c|c|c|c|c|c|}
\hline \multirow{3}{*}{$\frac{\text { Lines }}{\mathrm{V}_{1}}$} & \multicolumn{6}{|c|}{ Leaf area $\left(\mathrm{cm}^{2}\right)$ at 60 DAT } & \multicolumn{6}{|c|}{ Chlorophyll content (\%) at 60 DAT } \\
\hline & \multicolumn{2}{|c|}{ Control } & \multicolumn{2}{|c|}{$12 \mathrm{dS} / \mathrm{m}$} & \multicolumn{2}{|c|}{$16 \mathrm{dS} / \mathrm{m}$} & \multicolumn{2}{|c|}{ Control } & \multicolumn{2}{|c|}{$12 \mathrm{dS} / \mathrm{m}$} & \multicolumn{2}{|c|}{$16 \mathrm{dS} / \mathrm{m}$} \\
\hline & 109.9 & $\mathrm{~h}$ & 85.9 & $\mathrm{f}$ & 72.7 & de & 46.8 & efg & 38.1 & $\mathrm{e}$ & 33.2 & fgh \\
\hline $\mathrm{V}_{2}$ & 123.9 & $\mathrm{f}$ & 83.9 & $\mathrm{~g}$ & 73.1 & $\mathrm{~d}$ & 45.8 & fgh & 38.1 & $\mathrm{e}$ & 31.8 & ghi \\
\hline $\mathrm{V}_{3}$ & 157.7 & $b$ & 94.0 & $\mathrm{~d}$ & 68.9 & $\mathrm{~g}$ & 43.8 & $\mathrm{i}$ & 36.0 & $\mathrm{~g}$ & 31.1 & $\mathrm{i}$ \\
\hline $\mathrm{V}_{4}$ & 107.5 & $\mathrm{i}$ & 73.9 & $\mathrm{j}$ & 57.1 & $\mathrm{k}$ & 60.8 & $\mathrm{a}$ & 51.5 & $\mathrm{a}$ & 47.4 & $\mathrm{a}$ \\
\hline $\mathrm{V}_{5}$ & 128.0 & $\mathrm{e}$ & 89.9 & $\mathrm{e}$ & 70.0 & fg & 49.1 & $c$ & 41.9 & $\mathrm{c}$ & 37.9 & $c$ \\
\hline $\mathrm{V}_{6}$ & 128.1 & e & 91.1 & e & 80.2 & c & 58.8 & $\mathrm{~b}$ & 49.5 & $\mathrm{~b}$ & 43.7 & $\mathrm{~b}$ \\
\hline$V_{7}$ & 106.9 & $\mathrm{i}$ & 59.9 & $\mathrm{k}$ & 54.5 & 1 & 47.9 & cde & 40.5 & $\mathrm{~cd}$ & 35.8 & de \\
\hline $\mathrm{V}_{8}$ & 180.8 & $\mathrm{a}$ & 112.7 & b & 92.7 & b & 40.7 & $\mathrm{j}$ & 31.3 & $\mathrm{~h}$ & 26.8 & $\mathrm{j}$ \\
\hline $\mathrm{V}_{9}$ & 144.7 & $\mathrm{c}$ & 123.7 & $\mathrm{a}$ & 97.6 & $\mathrm{a}$ & 39.7 & $\mathrm{j}$ & 30.6 & $\mathrm{~h}$ & 25.6 & $\mathrm{j}$ \\
\hline $\mathrm{V}_{10}$ & 121.1 & $\mathrm{~g}$ & 72.1 & $\mathrm{j}$ & 61.0 & $\mathrm{j}$ & 47.0 & ef & 38.7 & de & 34.5 & def \\
\hline $\mathrm{V}_{11}$ & 97.9 & $\mathrm{j}$ & 79.0 & $\mathrm{i}$ & 62.1 & $\mathrm{ij}$ & 44.2 & hi & 35.8 & $\mathrm{~g}$ & 31.2 & $\mathrm{i}$ \\
\hline $\mathrm{V}_{12}$ & 130.1 & d & 81.0 & $\mathrm{~h}$ & 64.9 & $\mathrm{~h}$ & 47.2 & def & 38.9 & de & 34.0 & ef \\
\hline $\mathrm{V}_{13}$ & 130.8 & d & 84.6 & $\mathrm{fg}$ & 72.2 & de & 48.9 & $\mathrm{~cd}$ & 40.8 & $\mathrm{c}$ & 36.3 & $\mathrm{~cd}$ \\
\hline $\mathrm{V}_{14}$ & 123.9 & f & 83.9 & $\mathrm{~g}$ & 70.9 & ef & 46.4 & efg & 37.6 & efg & 33.4 & fg \\
\hline $\mathrm{V}_{15}$ & 131.0 & d & 97.8 & c & 71.7 & def & 46.0 & fgh & 37.9 & ef & 32.7 & fghi \\
\hline $\mathrm{V}_{16}$ & 111.0 & $\mathrm{~h}$ & 85.6 & $\mathrm{fg}$ & 62.9 & $\mathrm{i}$ & 44.9 & ghi & 36.1 & $\mathrm{fg}$ & 31.3 & hi \\
\hline LSD0.01 & 1.9 & & 1.9 & & 1.9 & & 1.9 & & 1.9 & & 1.9 & \\
\hline CV\% & 0.9 & & 1.3 & & 1.6 & & 2.4 & & 2.9 & & 3.3 & \\
\hline
\end{tabular}

${ }^{\mathrm{x}}$ In a column means having similar letter (s) are statistically identical and those having dissimilar letter (s) differ significantly as per 0.05 level of probability 


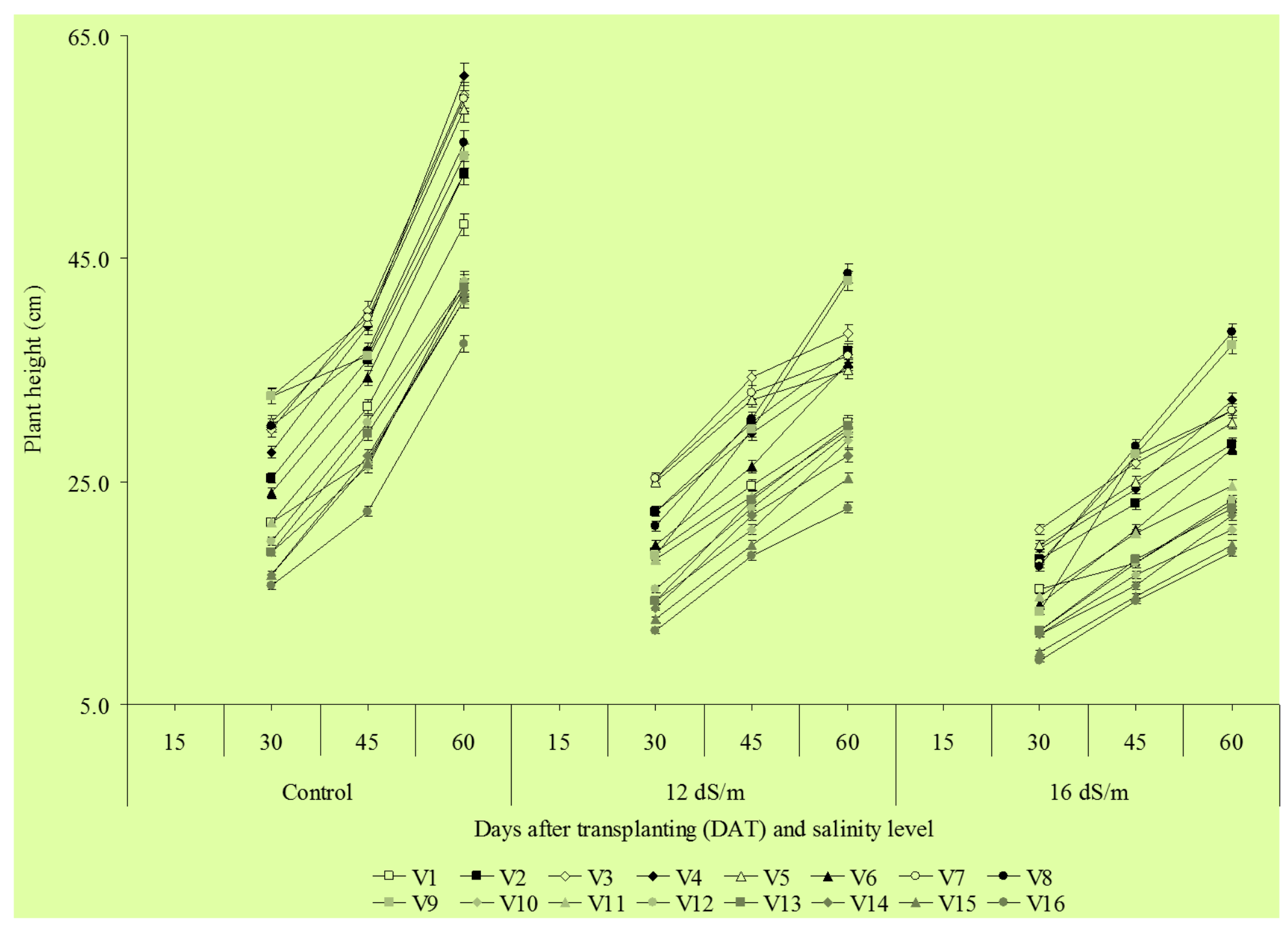

Figure 1. Response of 16 tomato varieties to plant height on 3 salinity levels at different days after transplanting 


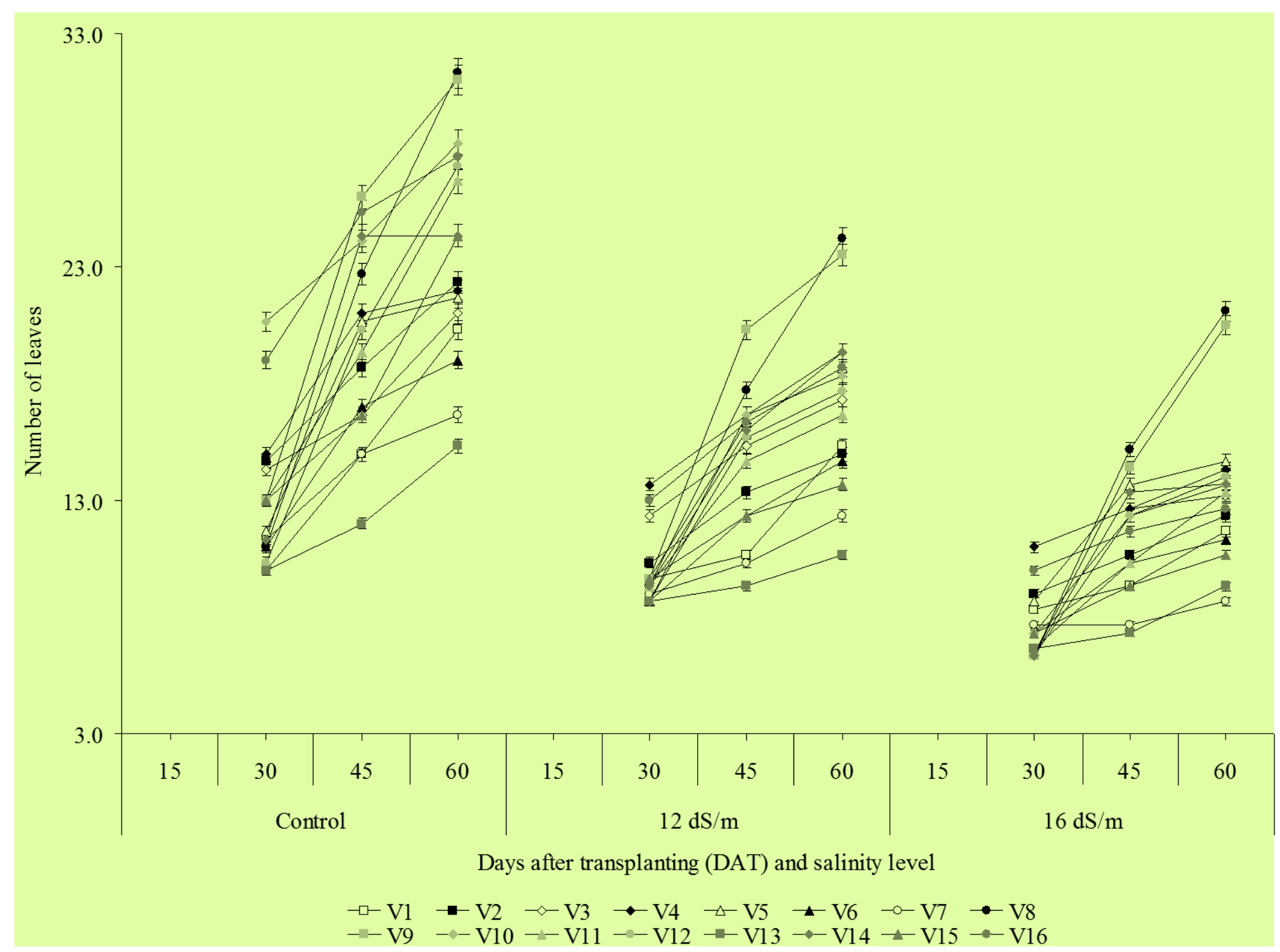

Figure 2. Response of 16 tomato lines to number of leaves on 3 salinity levels at different days after transplanting 


\section{Number of bunch per plant}

Maximum number of bunch per plant was found from $V_{8}$ (15.3) followed by $V_{9}$ (13.7) whereas minimum from $\mathrm{V}_{1}$ (7.7) in control (Table 1). On the other hand, maximum number of bunch/plant was observed from $\mathrm{V}_{9}$ (10.7 and 9.3 in $12 \mathrm{dS} / \mathrm{m}$ and $16 \mathrm{dS} / \mathrm{m}$ respectively) followed by $\mathrm{V}_{8}(9.3$ and 9.3 in $12 \mathrm{dS} / \mathrm{m}$ and $16 \mathrm{dS} / \mathrm{m}$ respectively) at different salinity levels (Table 2 ).

\section{Days to fruiting}

$\mathrm{V}_{3}$ was showed early fruiting (35.5, 26.7 and 22.7 days at control, $12 \mathrm{dS} / \mathrm{m}$ and $16 \mathrm{dS} / \mathrm{m}$ respectively) whereas late fruiting was found from $\mathrm{V}_{14}$ (50.7, 42.3 and 39.3 days at control, $12 \mathrm{dS} / \mathrm{m}$ and $16 \mathrm{dS} / \mathrm{m}$ respectively) (Table 2). From the findings of the present study it can be stated that tomato plant provided early fruiting when they were in saline stress.

Table 2. Number of bunch per plant and days to fruiting and of tomato lines at different salinity level $^{\mathrm{x}}$

\begin{tabular}{|c|c|c|c|c|c|c|c|c|c|c|c|c|}
\hline \multirow{3}{*}{$\begin{array}{r}\text { Lines } \\
\mathrm{V}_{1}\end{array}$} & \multicolumn{6}{|c|}{ Number of bunch per plant } & \multicolumn{6}{|c|}{ Days to fruit set } \\
\hline & \multicolumn{2}{|c|}{ Control } & \multicolumn{2}{|c|}{$12 \mathrm{dS} / \mathrm{m}$} & \multicolumn{2}{|c|}{$16 \mathrm{dS} / \mathrm{m}$} & \multicolumn{2}{|c|}{ Control } & \multicolumn{2}{|c|}{$12 \mathrm{dS} / \mathrm{m}$} & \multicolumn{2}{|c|}{$16 \mathrm{dS} / \mathrm{m}$} \\
\hline & 7.7 & $\mathrm{f}$ & 6.3 & efg & 5.3 & de & 41.3 & gh & 33.7 & $\mathrm{G}$ & 29.7 & $\mathrm{~g}$ \\
\hline $\mathrm{V}_{2}$ & 10.0 & $c d$ & 6.3 & efg & 5.0 & de & 44.0 & def & 36.3 & $\mathrm{E}$ & 32.3 & $\mathrm{e}$ \\
\hline $\mathrm{V}_{3}$ & 8.7 & def & 6.3 & efg & 4.7 & $\mathrm{e}$ & 35.3 & $\mathrm{i}$ & 26.7 & I & 22.7 & $\mathrm{j}$ \\
\hline $\mathrm{V}_{4}$ & 8.3 & ef & 7.0 & de & 5.3 & de & 45.0 & cde & 36.3 & $\mathrm{E}$ & 32.3 & e \\
\hline $\mathrm{V}_{5}$ & 9.7 & cde & 5.7 & $\mathrm{fg}$ & 4.3 & $\mathrm{e}$ & 43.3 & defgh & 33.7 & $\mathrm{G}$ & 29.7 & $\mathrm{~g}$ \\
\hline $\mathrm{V}_{6}$ & 9.7 & cde & 6.0 & efg & 4.3 & $\mathrm{e}$ & 42.3 & fgh & 35.3 & $\mathrm{~F}$ & 32.3 & $\mathrm{e}$ \\
\hline $\mathrm{V}_{7}$ & 10.0 & $\mathrm{~cd}$ & 8.0 & $\mathrm{~cd}$ & 6.3 & $\mathrm{~cd}$ & 45.3 & cde & 36.7 & $\mathrm{E}$ & 33.7 & $\mathrm{~d}$ \\
\hline $\mathrm{V}_{8}$ & 15.3 & $\mathrm{a}$ & 9.3 & $\mathrm{~b}$ & 8.3 & $a b$ & 45.7 & $\mathrm{~cd}$ & 39.3 & $\mathrm{C}$ & 34.3 & $\mathrm{c}$ \\
\hline $\mathrm{V}_{9}$ & 13.7 & b & 10.7 & $\mathrm{a}$ & 9.3 & $\mathrm{a}$ & 47.3 & $\mathrm{bc}$ & 36.7 & $\mathrm{E}$ & 30.7 & $\mathrm{f}$ \\
\hline $\mathrm{V}_{10}$ & 10.3 & $\mathrm{c}$ & 5.3 & $\mathrm{~g}$ & 4.3 & $\mathrm{e}$ & 41.0 & $\mathrm{~h}$ & 35.3 & $\mathrm{~F}$ & 28.3 & hi \\
\hline $\mathrm{V}_{11}$ & 9.7 & cde & 6.7 & ef & 4.3 & $\mathrm{e}$ & 42.3 & fgh & 32.7 & $\mathrm{H}$ & 26.7 & $\mathrm{i}$ \\
\hline $\mathrm{V}_{12}$ & 14.0 & $a b$ & 8.3 & $\mathrm{bc}$ & 6.3 & $\mathrm{~cd}$ & 43.0 & efgh & 35.3 & $\mathrm{~F}$ & 29.3 & $\mathrm{~g}$ \\
\hline $\mathrm{V}_{13}$ & 8.7 & def & 7.0 & de & 4.7 & $\mathrm{e}$ & 49.3 & $a b$ & 40.7 & B & 33.7 & d \\
\hline$V_{14}$ & 10.0 & $\mathrm{~cd}$ & 8.7 & $b c$ & 6.3 & $\mathrm{~cd}$ & 50.7 & $\mathrm{a}$ & 42.3 & A & 39.3 & $\mathrm{a}$ \\
\hline$V_{15}$ & 10.7 & $\mathrm{c}$ & 8.0 & $\mathrm{~cd}$ & 6.3 & $\mathrm{~cd}$ & 43.7 & defg & 38.7 & D & 34.7 & $\mathrm{c}$ \\
\hline$V_{16}$ & 15.0 & $a b$ & 8.3 & bc & 7.3 & bc & 43.7 & defg & 38.3 & D & 36.3 & b \\
\hline LSD0.01 & 1.4 & & 1.3 & & 1.6 & & 2.4 & & 0.5 & & 0.5 & \\
\hline CV\% & 7.9 & & 6.3 & & 6.7 & & 3.3 & & 0.8 & & 0.9 & \\
\hline
\end{tabular}

${ }^{\mathrm{x}}$ In a column means having similar letter (s) are statistically identical and those having dissimilar letter (s) differ significantly as per 0.05 level of probability

\section{Number of flower per plant and fruit per plant}

Number of flower per plant and fruit per plant varied significantly among the cultivars in different salinity levels. Maximum number of flower was found from $\mathrm{V}_{10}$ (76.3/palnt at control, 60.7/plant at $12 \mathrm{dS} / \mathrm{m}$ and 58.0/plant at $16 \mathrm{dS} / \mathrm{m})$ while minimum was in $\mathrm{V}_{7}$ at control and $12 \mathrm{dS} / \mathrm{m}(29.7 / \mathrm{plant}$ and 20.3/plant respectively) and $\mathrm{V}_{14}$ (9.0/plant) at $16 \mathrm{dS} / \mathrm{m}$ (Table 3). Maximum number of fruit was found from $\mathrm{V}_{8}$ (32.3/plant) followed by $\mathrm{V}_{9}$ (22.3) in control while maximum was found from $\mathrm{V}_{9}$ (20.3/plant and $16.3 /$ plant in $12 \mathrm{dS} / \mathrm{m}$ and $16 \mathrm{dS} / \mathrm{m}$ respectively) which was statistically similar with the $\mathrm{V}_{8}$ (19.3/plant and 16.3/plant in $12 \mathrm{dS} / \mathrm{m}$ and $16 \mathrm{dS} / \mathrm{m}$ respectively) (Table 3). 
Table 3. Number of flower and fruit per plant of tomato lines at different salinity level ${ }^{\mathrm{x}}$

\begin{tabular}{|c|c|c|c|c|c|c|c|c|c|c|c|c|}
\hline \multirow{3}{*}{$\begin{array}{c}\text { Lines } \\
\mathrm{V}_{1}\end{array}$} & \multicolumn{6}{|c|}{ No. of flower per plant } & \multicolumn{6}{|c|}{ Number of fruit per plant } \\
\hline & \multicolumn{2}{|c|}{ Control } & \multicolumn{2}{|c|}{$12 \mathrm{dS} / \mathrm{m}$} & \multicolumn{2}{|c|}{$16 \mathrm{dS} / \mathrm{m}$} & \multicolumn{2}{|c|}{ Control } & \multicolumn{2}{|c|}{$12 \mathrm{dS} / \mathrm{m}$} & \multicolumn{2}{|c|}{$16 \mathrm{dS} / \mathrm{m}$} \\
\hline & 46.3 & $\mathrm{~g}$ & 31.3 & fg & 36.3 & $\mathrm{~d}$ & 9.3 & $\mathrm{~h}$ & 8.3 & $\mathrm{~g}$ & 5.3 & fg \\
\hline $\mathrm{V}_{2}$ & 42.0 & $\mathrm{~h}$ & 28.3 & fgh & 25.3 & $\mathrm{f}$ & 10.3 & gh & 8.3 & $\mathrm{~g}$ & 7.3 & $\mathrm{e}$ \\
\hline $\mathrm{V}_{3}$ & 36.7 & $\mathrm{i}$ & 28.0 & gh & 30.0 & $\mathrm{e}$ & 9.3 & $\mathrm{~h}$ & 6.3 & h & 4.3 & g \\
\hline $\mathrm{V}_{4}$ & 36.3 & $\mathrm{i}$ & 30.7 & fgh & 20.3 & $\mathrm{~g}$ & 7.3 & $\mathrm{i}$ & 5.3 & $\mathrm{~h}$ & 4.3 & $\mathrm{~g}$ \\
\hline $\mathrm{V}_{5}$ & 42.7 & $\mathrm{~h}$ & 27.3 & $\mathrm{~h}$ & 12.3 & $\mathrm{~h}$ & 19.3 & $\mathrm{c}$ & 12.3 & $\mathrm{bc}$ & 7.3 & $\mathrm{e}$ \\
\hline $\mathrm{V}_{6}$ & 50.0 & $\mathrm{f}$ & 32.0 & ef & 26.7 & $\mathrm{f}$ & 16.3 & d & 9.3 & efg & 6.3 & ef \\
\hline $\mathrm{V}_{7}$ & 29.7 & $\mathrm{j}$ & 20.3 & $\mathrm{i}$ & 15.3 & h & 12.3 & $\mathrm{f}$ & 8.3 & $\mathrm{~g}$ & 7.3 & $\mathrm{e}$ \\
\hline $\mathrm{V}_{8}$ & 37.0 & $\mathrm{i}$ & 27.3 & $\mathrm{~h}$ & 20.3 & $\mathrm{~g}$ & 32.3 & $\mathrm{a}$ & 19.3 & $\mathrm{a}$ & 16.3 & $\mathrm{a}$ \\
\hline $\mathrm{V}_{9}$ & 43.0 & $\mathrm{~h}$ & 35.7 & $\mathrm{e}$ & 30.7 & $\mathrm{e}$ & 22.3 & b & 20.3 & $\mathrm{a}$ & 16.3 & $\mathrm{a}$ \\
\hline $\mathrm{V}_{10}$ & 76.3 & $\mathrm{a}$ & 60.7 & $\mathrm{a}$ & 58.0 & $\mathrm{a}$ & 20.3 & $\mathrm{c}$ & 9.3 & efg & 7.3 & $\mathrm{e}$ \\
\hline $\mathrm{V}_{11}$ & 66.0 & b & 40.0 & $\mathrm{~d}$ & 49.7 & c & 17.3 & $\mathrm{~d}$ & 13.3 & $\mathrm{~b}$ & 14.3 & b \\
\hline $\mathrm{V}_{12}$ & 56.0 & d & 49.7 & $\mathrm{~b}$ & 50.7 & $\mathrm{bc}$ & 13.3 & $\mathrm{f}$ & 10.3 & de & 9.3 & d \\
\hline $\mathrm{V}_{13}$ & 36.7 & $\mathrm{i}$ & 29.3 & fgh & 53.0 & $\mathrm{~b}$ & 10.0 & gh & 9.0 & $\mathrm{fg}$ & 9.0 & d \\
\hline $\mathrm{V}_{14}$ & 53.7 & $\mathrm{e}$ & 46.7 & $\mathrm{bc}$ & 9.0 & $\mathrm{i}$ & 11.0 & $\mathrm{~g}$ & 6.0 & $\mathrm{~h}$ & 5.0 & $\mathrm{~g}$ \\
\hline $\mathrm{V}_{15}$ & 60.0 & $\mathrm{c}$ & 44.7 & $\mathrm{c}$ & 14.0 & $\mathrm{~h}$ & 17.3 & $\mathrm{~d}$ & 11.3 & $\mathrm{~cd}$ & 4.3 & $\mathrm{~g}$ \\
\hline $\mathrm{V}_{16}$ & 52.3 & $\mathrm{e}$ & 47.3 & $\mathrm{bc}$ & 37.0 & $\mathrm{~d}$ & 15.0 & $\mathrm{e}$ & 10.0 & ef & 12.0 & $\mathrm{c}$ \\
\hline LSD0.01 & 2.1 & & 3.7 & & 3.1 & & 1.0 & & 1.0 & & 1.0 & \\
\hline $\mathrm{CV} \%$ & 2.7 & & 6.1 & & 6.0 & & 4.1 & & 6.0 & & 7.3 & \\
\hline
\end{tabular}

${ }^{\mathrm{x}} \mathrm{In}$ a column means having similar letter (s) are statistically identical and those having dissimilar letter (s) differ significantly as per 0.05 level of probability

Table 4. Single fruit weight and yield/plant of tomato lines at different salinity level ${ }^{\mathrm{x}}$

\begin{tabular}{|c|c|c|c|c|c|c|c|c|c|c|c|c|}
\hline \multirow{3}{*}{$\begin{array}{c}\text { Lines } \\
\mathrm{V}_{1}\end{array}$} & \multicolumn{6}{|c|}{ Single fruit weight (g) } & \multicolumn{6}{|c|}{ Yield $(\mathrm{kg}) /$ plant } \\
\hline & \multicolumn{2}{|c|}{ Control } & \multicolumn{2}{|c|}{$12 \mathrm{dS} / \mathrm{m}$} & \multicolumn{2}{|c|}{$16 \mathrm{dS} / \mathrm{m}$} & \multicolumn{2}{|c|}{ Control } & \multicolumn{2}{|c|}{$12 \mathrm{dS} / \mathrm{m}$} & \multicolumn{2}{|l|}{$16 \mathrm{dS} / \mathrm{m}$} \\
\hline & 65.3 & $\mathrm{f}$ & 43.3 & $\mathrm{c}$ & 30.4 & $\mathrm{c}$ & 0.627 & o & 0.373 & $\mathrm{e}$ & 0.183 & $\mathrm{~g}$ \\
\hline $\mathrm{V}_{2}$ & 76.4 & $c$ & 36.9 & $\mathrm{e}$ & 26.3 & $\mathrm{e}$ & 0.793 & $\mathrm{i}$ & 0.323 & $\mathrm{f}$ & 0.213 & $\mathrm{f}$ \\
\hline $\mathrm{V}_{3}$ & 130.5 & $\mathrm{a}$ & 35.9 & $f$ & 16.9 & $\mathrm{~h}$ & 1.193 & $\mathrm{~b}$ & 0.243 & $\mathrm{i}$ & 0.137 & $\mathrm{k}$ \\
\hline $\mathrm{V}_{4}$ & 90.5 & b & 22.9 & 1 & 22.5 & $\mathrm{f}$ & 0.663 & $\mathrm{k}$ & 0.143 & o & 0.157 & $\mathrm{i}$ \\
\hline $\mathrm{V}_{5}$ & 42.3 & $\mathrm{n}$ & 16.9 & o & 15.3 & $\mathrm{i}$ & 0.833 & g & 0.233 & $\mathrm{j}$ & 0.143 & $\mathrm{j}$ \\
\hline $\mathrm{V}_{6}$ & 42.4 & $\mathrm{n}$ & 29.0 & $\mathrm{~g}$ & 9.2 & 1 & 0.647 & 1 & 0.227 & $\mathrm{k}$ & 0.050 & o \\
\hline$V_{7}$ & 71.5 & d & 23.6 & $\mathrm{k}$ & 8.5 & $\mathrm{~m}$ & 0.893 & f & 0.223 & 1 & 0.127 & 1 \\
\hline $\mathrm{V}_{8}$ & 64.4 & $\mathrm{~g}$ & 24.2 & $\mathrm{j}$ & 21.3 & g & 2.093 & $\mathrm{a}$ & 0.493 & b & 0.407 & b \\
\hline $\mathrm{V}_{9}$ & 46.5 & $\mathrm{~m}$ & 44.5 & $\mathrm{~b}$ & 35.3 & $\mathrm{a}$ & 1.053 & $\mathrm{e}$ & 0.923 & $\mathrm{a}$ & 0.593 & $\mathrm{a}$ \\
\hline $\mathrm{V}_{10}$ & 51.5 & $\mathrm{k}$ & 40.4 & d & 34.7 & b & 1.063 & d & 0.413 & d & 0.273 & d \\
\hline $\mathrm{V}_{11}$ & 35.4 & o & 20.4 & $\mathrm{~m}$ & 14.4 & $\mathrm{j}$ & 0.633 & $\mathrm{n}$ & 0.293 & $\mathrm{~h}$ & 0.233 & e \\
\hline $\mathrm{V}_{12}$ & 59.3 & $\mathrm{~h}$ & 27.3 & h & 29.5 & d & 0.803 & h & 0.303 & $\mathrm{~g}$ & 0.293 & $\mathrm{c}$ \\
\hline $\mathrm{V}_{13}$ & 54.4 & $\mathrm{j}$ & 48.5 & $\mathrm{a}$ & 14.4 & $\mathrm{j}$ & 0.573 & $\mathrm{p}$ & 0.463 & $\mathrm{c}$ & 0.163 & $\mathrm{~h}$ \\
\hline $\mathrm{V}_{14}$ & 55.4 & $\mathrm{i}$ & 25.6 & $\mathrm{i}$ & 15.4 & $\mathrm{i}$ & 0.643 & $\mathrm{~m}$ & 0.183 & $\mathrm{n}$ & 0.113 & $\mathrm{n}$ \\
\hline $\mathrm{V}_{15}$ & 66.5 & e & 19.5 & $\mathrm{n}$ & 3.4 & $\mathrm{n}$ & 1.163 & c & 0.243 & $\mathrm{i}$ & 0.043 & $\mathrm{p}$ \\
\hline $\mathrm{V}_{16}$ & 48.4 & 1 & 20.3 & $\mathrm{~m}$ & 9.9 & $\mathrm{k}$ & 0.720 & $\mathrm{j}$ & 0.200 & $\mathrm{~m}$ & 0.120 & $\mathrm{~m}$ \\
\hline LSD0.01 & 0.2 & & 0.2 & & 0.2 & & 0.002 & & 0.002 & & 0.002 & \\
\hline CV\% & 0.2 & & 0.3 & & 0.5 & & 1.560 & & 2.650 & & 2.840 & \\
\hline
\end{tabular}

${ }^{\mathrm{x}}$ In a column means having similar letter (s) are statistically identical and those having dissimilar letter (s) differ significantly as per 0.05 level of probability

From the result of the current study (Table 3), it was observed that $\mathrm{V}_{10}$ provided the maximum number of flower in control, $12 \mathrm{dS} / \mathrm{m}$ and $16 \mathrm{dS} / \mathrm{m}$ but maximum number of fruit was found from $\mathrm{V}_{8}$ and $\mathrm{V}_{9}$ in control, $12 \mathrm{dS} / \mathrm{m}$ and $16 \mathrm{dS} / \mathrm{m}$. Though $\mathrm{V}_{10}$ were able to produce maximum flower but they were not able to set maximum number of fruit not only in salinity stress but also in control condition. 


\section{Single fruit weight and yield per plant}

Single fruit weight and yield per plant showed significant variation among the variety at different salinity levels. Maximum single fruit weight was found from $\mathrm{V}_{3}(130.5 \mathrm{~g})$ in control, $\mathrm{V}_{13}(48.5 \mathrm{~g}) 12$ $\mathrm{dS} / \mathrm{m}$ and $\mathrm{V}_{9}(35.3 \mathrm{~g})$ in $16 \mathrm{dS} / \mathrm{m}$ (Table 4). However, maximum yield/plant was recorded from $\mathrm{V}_{8}$ $(2.093 \mathrm{~kg})$ followed by $\mathrm{V}_{3}(1.193 \mathrm{~kg})$ in control (Table 4). In case of different salinity level maximum yield was found from $\mathrm{V}_{9}(0.923 \mathrm{~kg} / \mathrm{plant}$ in $12 \mathrm{dS} / \mathrm{m}$ and $0.593 \mathrm{~kg} / \mathrm{plant}$ in $16 \mathrm{dS} / \mathrm{m})$ followed by $\mathrm{V}_{8}$ $(0.493 \mathrm{~kg} /$ plant in $12 \mathrm{dS} / \mathrm{m}$ and $0.407 \mathrm{~kg} /$ plant in $16 \mathrm{dS} / \mathrm{m})($ Table 4$)$.

\section{Best varieties at $12 \mathrm{dS} / \mathrm{m}$ and $16 \mathrm{dS} / \mathrm{m}$ salinity level}

At $12 \mathrm{dS} / \mathrm{m}$ salinity level, maximum no of fruit was found from $\mathrm{V}_{9}(20.3)$ followed by $\mathrm{V}_{8}(19.3)$ (Table 5). Maximum single fruit weight was found from $\mathrm{V}_{13}(48.5 \mathrm{~g})$ which was followed by $\mathrm{V}_{9}(44.5$ g) (Table 5). On the other hand, maximum yield/plant was found from $\mathrm{V}_{9}(0.923 \mathrm{~kg})$ which was followed $\mathrm{V}_{2}(0.493 \mathrm{~kg})$ (Table 6). At $16 \mathrm{dS} / \mathrm{m}$ salinity level, maximum no of fruit was found from $\mathrm{V}_{8}$ and $V_{9}$ (16.3) followed by $V_{11}$ (14.3) (Table 5). Maximum single fruit weight was found from $V_{9}$ $(35.3 \mathrm{~g})$ which was followed by $\mathrm{V}_{10}(34.7 \mathrm{~g})$ (Table 5). On the other hand, maximum yield/plant was provided by $\mathrm{V}_{9}(0.593 \mathrm{~kg})$ which was followed by $\mathrm{V}_{8}(0.407 \mathrm{~kg})$ (Table 5$)$.

Table 5. Performance of best tomato lines under salinity in yield related attributes

\begin{tabular}{|c|c|c|c|c|c|}
\hline \multicolumn{6}{|c|}{ At $12 \mathrm{dS} / \mathrm{m}$} \\
\hline Lines & $\begin{array}{l}\text { No. of } \\
\text { fruit/plant }\end{array}$ & Lines & $\begin{array}{l}\text { Single fruit } \\
\text { weight }(\mathrm{g})\end{array}$ & Lines & $\begin{array}{c}\text { Yield } \\
(\mathrm{kg}) / \text { plant }\end{array}$ \\
\hline $\mathrm{V}_{9}$ & 20.3 & $\mathrm{~V}_{13}$ & 48.5 & $\mathrm{~V}_{9}$ & 0.923 \\
\hline $\mathrm{V}_{8}$ & 19.3 & $\mathrm{~V}_{9}$ & 44.5 & $\mathrm{~V}_{2}$ & 0.493 \\
\hline \multicolumn{6}{|c|}{ At $16 \mathrm{dS} / \mathrm{m}$} \\
\hline Lines & $\begin{array}{l}\text { No. of } \\
\text { fruit/plant }\end{array}$ & Lines & $\begin{array}{l}\text { Single fruit } \\
\text { weight }(\mathrm{g})\end{array}$ & Lines & $\begin{array}{c}\text { Yield } \\
(\mathrm{kg}) / \text { plant }\end{array}$ \\
\hline $\begin{array}{c}\mathrm{V}_{9} \text { and } \mathrm{V}_{8} \\
\mathrm{~V}_{11}\end{array}$ & $\begin{array}{l}16.3 \\
14.3\end{array}$ & $\begin{array}{l}\mathrm{V}_{9} \\
\mathrm{~V}_{10}\end{array}$ & $\begin{array}{l}35.3 \\
34.7\end{array}$ & $\begin{array}{l}\mathrm{V}_{9} \\
\mathrm{~V}_{8}\end{array}$ & $\begin{array}{l}0.593 \\
0.407\end{array}$ \\
\hline
\end{tabular}

\section{Reduction percentage of number of fruits, single fruit weight and yield per plant}

Number of fruit was reduced $9.0-54.1 \%$ at $12 \mathrm{dS} / \mathrm{m}$ and $10.0-75.0 \%$ at $16 \mathrm{dS} / \mathrm{m}$ over control among the tomato lines (Table 6). Maximum number of the fruit was found from $V_{9}(20.3)$ and $V_{8}(19.3)$ at $12 \mathrm{dS} / \mathrm{m}$ while $\mathrm{V}_{9}(16.3), \mathrm{V}_{8}(16.3)$ and $\mathrm{V}_{11}(14.3)$ at $16 \mathrm{dS} / \mathrm{m}$ (Table 5) but number of fruit decreased $9.0 \%$ in $\mathrm{V}_{9}$ and $40.2 \%$ in $\mathrm{V}_{8}$ at $12 \mathrm{dS} / \mathrm{m}$ (Table 6) whereas $26.9 \%$ in $\mathrm{V}_{9}, 49.5 \%$ in $\mathrm{V}_{8}$ and $17.3 \%$ in $\mathrm{V}_{11}$ at $16 \mathrm{dS} / \mathrm{m}$ over control (Table 6).

Single fruit weight was decreased $4.3-74.7 \%$ at $12 \mathrm{dS} / \mathrm{m}$ and $24.1-94.9 \%$ at $16 \mathrm{dS} / \mathrm{m}$ over control among tomato lines (Table 6). Maximum single fruit weight was found from $\mathrm{V}_{13}(48.5 \mathrm{~g})$ and $\mathrm{V}_{9}(44.5$ g) at $12 \mathrm{dS} / \mathrm{m}$ whereas $\mathrm{V}_{9}(35.3 \mathrm{~g})$ and $\mathrm{V}_{10}(34.7 \mathrm{~g})$ at $16 \mathrm{dS} / \mathrm{m}$ (Table 5) but the single fruit weight was decreased $10.8 \%$ in $\mathrm{V}_{13}$ and $4.3 \%$ in $\mathrm{V}_{9}$ at $12 \mathrm{dS} / \mathrm{m}$ while $24.1 \%$ in $\mathrm{V}_{9}$ and $32.6 \%$ in $\mathrm{V}_{10}$ at 16 $\mathrm{dS} / \mathrm{m}$ over control (Table 6).

Yield per plant was also decreased due to the increases of salinity level. Decreases of the yield ranged from $12.3-79.6 \%$ at $12 \mathrm{dS} / \mathrm{m}$ and $43.7-96.3 \%$ at $1 \mathrm{dS} / \mathrm{m}$ over control (Table 6). Maximum yield per plant was found from $\mathrm{V}_{9}(0.923 \mathrm{~kg})$ and $\mathrm{V}_{2}(0.493 \mathrm{~kg})$ at $12 \mathrm{dS} / \mathrm{m}$ while $\mathrm{V}_{9}(0.593 \mathrm{~kg})$ and $\mathrm{V}_{8}(0.407$ $\mathrm{kg}$ ) at $16 \mathrm{dS} / \mathrm{m}$ (Table 5) but single fruit weight was decreased $12.3 \%$ in $\mathrm{V}_{9}$ and $59.2 \%$ in $\mathrm{V}_{2}$ whereas $43.7 \%$ in $\mathrm{V}_{9}$ and $80.6 \%$ in $\mathrm{V}_{8}$ over control (Table 6). 
Table 6. Reduction percentage of number of fruits, single fruit weight and yield per plant at salinity level compared to the control

\begin{tabular}{|c|c|c|c|c|c|c|}
\hline \multirow{3}{*}{ Lines } & \multicolumn{6}{|c|}{ Reduction (\%) } \\
\hline & \multicolumn{2}{|c|}{ Number of fruits } & \multicolumn{2}{|c|}{ Single fruit weight } & \multicolumn{2}{|c|}{ Yield $(\mathrm{kg}) /$ plant } \\
\hline & $12 \mathrm{dS} / \mathrm{m}$ & $16 \mathrm{dS} / \mathrm{m}$ & $12 \mathrm{dS} / \mathrm{m}$ & $16 \mathrm{dS} / \mathrm{m}$ & $12 \mathrm{dS} / \mathrm{m}$ & $16 \mathrm{dS} / \mathrm{m}$ \\
\hline $\mathrm{V}_{1}$ & 10.7 & 42.9 & 33.7 & 53.4 & 40.4 & 70.8 \\
\hline $\mathrm{V}_{2}$ & 19.3 & 29.0 & 51.7 & 65.6 & 59.2 & 73.1 \\
\hline $\mathrm{V}_{3}$ & 32.1 & 53.6 & 72.5 & 87.0 & 79.6 & 88.5 \\
\hline $\mathrm{V}_{4}$ & 27.3 & 40.9 & 74.7 & 75.1 & 78.4 & 76.4 \\
\hline $\mathrm{V}_{5}$ & 36.2 & 62.1 & 60.0 & 63.8 & 72.0 & 82.8 \\
\hline $\mathrm{V}_{6}$ & 42.8 & 61.2 & 31.6 & 78.4 & 64.9 & 92.3 \\
\hline $\mathrm{V}_{7}$ & 32.4 & 40.5 & 67.0 & 88.1 & 75.0 & 85.8 \\
\hline $\mathrm{V}_{8}$ & 40.2 & 49.5 & 62.4 & 66.9 & 76.4 & 80.6 \\
\hline $\mathrm{V}_{9}$ & 9.0 & 26.9 & 4.3 & 24.1 & 12.3 & 43.7 \\
\hline $\mathrm{V}_{10}$ & 54.1 & 63.9 & 21.6 & 32.6 & 61.1 & 74.3 \\
\hline $\mathrm{V}_{11}$ & 23.1 & 17.3 & 42.3 & 59.3 & 53.7 & 63.2 \\
\hline $\mathrm{V}_{12}$ & 22.5 & 30.0 & 54.0 & 50.3 & 62.2 & 63.5 \\
\hline $\mathrm{V}_{13}$ & 10.0 & 10.0 & 10.8 & 73.5 & 19.2 & 71.5 \\
\hline $\mathrm{V}_{14}$ & 45.5 & 54.5 & 53.8 & 72.2 & 71.5 & 82.4 \\
\hline $\mathrm{V}_{15}$ & 34.6 & 75.0 & 70.7 & 94.9 & 79.1 & 96.3 \\
\hline $\mathrm{V}_{16}$ & 33.3 & 20.0 & 58.1 & 79.5 & 72.2 & 83.3 \\
\hline
\end{tabular}

\section{Discussion}

A successful salt tolerant cultivar should exhibit salt tolerance without compromising its yield potential. Therefore some characters of tomato plant have to be taken into consideration in this study. The response of tomato cultivars to increasing salinity levels as $12 \mathrm{dS} / \mathrm{m}$ and $16 \mathrm{dS} / \mathrm{m}$ in which a significant decrease in plant height, leaves number/plant, leaf area, chlorophyll content, number of bunches, number of flowers/plant, number of fruits/plant, single fruit weight and yield was seen as the salt concentration increased (Fig. 1-2 and Table 1-4). Plant leaf number was reduced by $23.3 \%$ and average reduction of plant height was $29.03 \%$ with the salinity (Oztekin and Tuzel, 2011). Salt stress affects the plant growth and development thereby affecting the yield quantity and quality (Foolad, 2004; Maggio et al., 2002; Cuartero et al., 2006; Sattar et al., 2010). Previous studies denotes that a reduced growth at low concentrations of salt is caused by less availability of nutrients require for its growth. As salt concentration increases besides nutrient imbalance, hyperomostic stress and ion disequilibrium plays a pivotal role in disturbing the cellular functions of plant (Foolad, 2004). In developing salt tolerant tomato cultivars, heritability of the selected trait has to be considered along with its physiological and metabolic importance. Leaf area showed the highest heritability as compared to shoot dry weight, measures of ion contents and water relations (Cuartero et al., 2006). Present study showed significant decrease in leaf area of tomato leaves with application elevated salt treatment (Table 1). Salt stress reduced leaf growth rate by shortening the length of the leaf elongating zone and decreasing the growth intensity in its central and distal portions (Bernstein et al., 1993). Leaf growth inhibition by salinity must be expected to occur via an effect on this region (Lazof and Bernstein, 1998). Under saline condition as soon as new cell starts its elongation process, the excess of $\mathrm{Na}^{+}, \mathrm{Cl}^{-}$and other ions modifies the metabolic activities of cell wall, which causes deposition of several materials on cell wall and limits the cell wall elasticity (Yasar et al., 2006). Cell walls become rigid and turgor pressure efficiency in cell enlargement is decreased with application of elevated salt treatment. The other anticipated cause of reduction in leaf area and dry matter content could be the reduced development and differentiation of tissues, shrinkage of the cell contents, unbalanced nutrition, damage of membrane and disturbed avoidance mechanism (Akram et al. 2007). Plant has a reduction in its growth due to the proportional increase of Na. High salinity may inhibit root and shoot 
elongation due to the lower water uptake by the plant (Werner and Finkelstein, 1995). Root growth was more adversely affected as compared to shoot growth by salinity (Demir and Arif, 2003). From the current experiment it was found that significant decrease in leaf area of tomato leaves with application elevated salt treatment (Table 1).

Chlorophyll content decreases in salt susceptible plants such as tomato (Lapina and Popov, 1970). $\mathrm{NaCl}$ stress decreased total chlorophyll content of plant by increasing the activity of the chlorophyll degrading enzyme: cholorophyllase (Rao and Rao, 1981), inducing the destruction of the chloroplast structure and instability of pigment protein complexes (Singh and Dubey, 1995). At the highest salinity level $(9.6 \mathrm{dS} / \mathrm{m})$, the plant growth traits were smaller than those at the control level $(0.5 \mathrm{dS} / \mathrm{m})$ by approximately 13,11,17, 16 and $18 \%$ for plant height, stem diameter, leaf area, leaf fresh weight and dry weight, respectively. All of the plant growth traits responded similarly to salinity that is the indication of effect salt of stress on allocation and distribution of photosynthetic resources within various plant organs (Alsadon et al., 2013; Olympios et al., 2003). Early flowering and decreases the number of fruit, single fruit weight and yield caused by increasing the salinity level (Table 2-4). Average fruit weight of tomato plant reduced due to the increase of salinity level (Table 6). The successive increase in salinity level from 0.5 to $2.4,4.8,7.2$ and $9.6 \mathrm{dS} / \mathrm{m}$ caused a reduction in average fruit weight by $7,18,27$, and $31 \%$, respectively, compared with the control but reductions in the number of fruit per plant were $2,5,8$ and $10 \%$ for $2.4,4.8,7.2$ and $9.6 \mathrm{dS} / \mathrm{m}$, the total yield was reduced by 4, 18, 25 and 31\%, respectively (Alsadon et al., 2013). Enhanced early growth of tomato with the intermediate salinity treatment may have been due to the addition of plant nutrients to irrigation water (Bolarin et al., 1993) and drastic decrease in fruit size (Vinten et al., 1986).

Means of different genotypes over all levels of salinity demonstrated a wide range of variability in average fruit weight, fruit number and total yield (Tables 3-4). The decrease in total yield can be ascribed to the significant decrease in fruit number than the decrease in single fruit weight because the reduction in fruit number was greater than that single fruit weight (Table 3-4). The effect of salinity on yield became more marked as the harvest period progressed, initially because of a restriction of fruit size and later because of a decrease in fruit number (Cuartero and Fernandez-Munnza, 1999). Salt stress is an abiotic stress factor that causes various deleterious effects on the overall plant growth and development (Ghanem et al., 2008). There exist several internal and external factors that influence the overall growth and development of plants. Plant growth hormones, which are the signaling molecules helps in transmitting signals between the cells and within the cells thus aids in development of plants in each and every stage of their life is one such internal factor. External factors include water relations, proper nutrient supplies etc. All these above factors such as water relations, carbon supply, hormonal balance involved in the control of growth of plants exposed to high salt concentrations (Albacete et al., 2008). Salt stress causes extensive physiological and biochemical changes in plant. Between these changes, distribution in different organs of the plant's intake of ions like $\mathrm{K}^{+}, \mathrm{Ca}^{+2}$ and $\mathrm{Na}^{+}$is emphasized (Aziz et al., 1999). In conditions which salt concentration is high, the plant gets more $\mathrm{Na}^{+}$ion than it needs (Levitt, 1980). Exposure of plants to $\mathrm{NaCl}$ reduced the availability of water for the plants, thus causes osmotic stress. The dominance of $\mathrm{Na}+$ and $\mathrm{Cl}$ - ions inhibits the uptake of other minerals vital for plant's growth (Ghanem et al., 2008; Albacete et al., 2008; Hamdia and Shaddad, 2010). Concentration of hormones such as auxin, cytokinin and zeatin changes in response to salinity (Ghanem et al., 2008, Albacete et al., 2008). This hormonal imbalance, osmotic stress etc is believed to play a significant role in the salinity induced changes in the shoot vigour and yield of plants (Albacete et al., 2008). Comparing the yield at high salinity level $(12.0$ and $16.0 \mathrm{dS} / \mathrm{m})$ with those at the control level $(0.0 \mathrm{dS} / \mathrm{m})$ the results indicate that the two salttolerant breeding lines $\mathrm{V}_{9}$ and $\mathrm{V}_{2}$ had the lowest reduction for yield in $12.0 \mathrm{dS} / \mathrm{m}$ and $\mathrm{V}_{9}$ and $\mathrm{V}_{8}$ had the lowest reduction for yield in $16.0 \mathrm{dS} / \mathrm{m}$ (Table 5). These result indicated that $\mathrm{V}_{9}$ (for both 12.0 and $16.0 \mathrm{dS} / \mathrm{m}$ ), $\mathrm{V}_{2}$ (for $12.0 \mathrm{dS} / \mathrm{m}$ ) and $\mathrm{V}_{8}$ (for $16.0 \mathrm{dS} / \mathrm{m}$ ) lines can be classified as salt tolerant. 


\section{Conclusion}

Salt tolerance in crops is largely determined by their ability to exclude $\mathrm{Na}^{+}$and $\mathrm{Cl}^{-}$from their shoots and their ability to maintain these ions. From the results it can be concluded that different levels of salinity significantly affect the performance of different tomato lines. With the increase of salinity levels the growth and yield decrease and these effect of differs among the lines. Plant growth and yield contributing characteristics also yield were changed to different according lines. However, $\mathrm{V}_{9}$ was best variety for the both the level of $12 \mathrm{dS} / \mathrm{m}$ and $16 \mathrm{dS} / \mathrm{m}$ saline affected area that was closely followed to the $\mathrm{V}_{8}$ concerning yield and yield contributing characters.

\section{References}

Akram, M., Malik, M. A., Ashraf, M. Y., Saleem, M. F. \& Hussain, M. (2007). Competitive Seedling Growth and $\mathrm{K}^{+} / \mathrm{Na}^{+}$Ratio in Different Maize (Zea mays L.) Hybrids under Salinity Stress. Pakistan J. Bot., 39: 2553-2563.

Albacete, A., Ghanem, M. E., Martinez-Andujar, C., Acosta, M., Sanchez-Bravo, J., Martinez, V., Lutts, S., Dodd, I. C. \& Perez-Alfocea, F. (2008). Hormonal changes in relation to biomass partitioning and shoot growth impairment in salinized tomato (Solanum lycopersicum L.) plants. J. Exp. Bot., 59: 4119-4131.

Allen, R. G., Pereira, L. S., Raes, D. \& Smith, M. (1998). Crop evapotranspiration: Guidelines for computing crop water requirements. Irrigation \& Drainage. Paper 56. UN-FAO, Rome, Italy.

Alsadon, A., Sadder, M. \& Wahb-Allah, M. (2013). Responsive gene screening and exploration of genotypes responses to salinity tolerance in tomato. Australian J. of Crop Sci., 7(9): 13831395.

Ashraf, M. \& McNeilly, T. (2004). Salinity tolerance in Brassica oilseeds. Critical Review of Plant Science, 23(2): 157-174.

Ashraf, M. \& Wahed, A. (1993). Response of some genetically divers lines of chickpea to salt. Australian J. Plant Physiol., 154: 257-266.

Aziz, A., Martin-Tanguay, J. \& Larher, F. (1999). Salt stress- induced proline accumulation and changes in tyramine and polyamine levels are linked to ionic adjustment tomato leafs discs. Plant Science, Elsevier Science Ireland Ltd. pp. 27-31

Bernstein, N., Lauchli, A. \& Silk, W. K. 1993. Kinematics and dynamics of sorghum (Sorghum bicolor L.) leaf development at various $\mathrm{Na} / \mathrm{Ca}$ salinities: I. Elongation growth. Plant Physiology, 103: 1107-1114.

Bolarin, M. C., Perez-Alfacea, F., Cano, E. A., Estan, M. T. \& Caro, M. (1993). Growth, fruit yield, and ion concentration in tomato genotypes after pre- and post-emergence salt treatments. $J$. Amer. Soc. Hort. Sci., 118: 655-660.

Caro, M., Cruz, V., Cuartero, J., Estan, M. T., and Bolarin, M. C. (1991). Salinity tolerance of normal-fruited and cherry tomato cultivars. Plant and Soil, 136: 249- 255.

Cuartero, J. \& Fernandez-Munoz, R. (1999). Tomato and salinity. Sci. Hort., 78: 83-125.

Cuartero, J., Bolarin, M. C., Asins, M. J. \& Moreno, V. (2006). Increasing salt tolerance in tomato. J. Exp. Bot., 57: 1045-1058.

Demir, M. \& Arif, I. (2003). Effects of different soil salinity levels on germination and seedling growth of safflower (Carthamus tinctoriusl). Turkish J. of Agriculture, 27: 221-227.

Foolad, M. R. (2004). Recent advances in genetics of salt tolerance in tomato. Plant and Organ Culture, 76: 101-119. 
Ghanem, M. E., Albacete, A., Martínez-Andujar, C., Acosta, M., Romero-Aranda, R., Dodd, I. C., Lutts, S. \& Perez-Alfocea, F. (2008). Hormonal changes during salinity-induced leaf senescence in tomato (Solanum lycopersicum L.). J. Exp. Bot., 59: 3039-3050.

Ghanem, M. E., Albacete, A., Smigocki, A. C., Frebort, I. \& Pospisilova, H. (2011). Rootsynthesized cytokinins improve shoot growth and fruit yield in salinized tomato (Solanum lycopersicum L.). Plants J. Exp. Bot., 62: 125-140.

Gomez, K. A. \& Gomez, A. A. (1984). Statistical Procedures for Agricultural Research. $2^{\text {nd }}$ edn. John Wiley and Sons. New York. 680.

Hajiboland, R., Joudmand, A. \& Fotouhi, K. (2009). Mild salinity improves sugar beet (Beta vulgaris L.) quality. Acta Agri. Scand B: Soil Plant Sci., 59: 295-305.

Hamdia, M. A. \& Shaddad, M. A. K. (2010). Salt tolerance of crop plants. J. Stress Physiol. Biochem., 6: 64-90.

Karlberg, L., Ben-Gal, A., Jansson, P. E. \& Shani, U. (2006). Modeling transpiration and growth in salinity-stressed tomato under different climatic conditions. Ecological Modeling, 190, 15-40.

Lapina, L. P. \& Popov, B. A. (1970). Effect of sodium chloride on photosynthetic apparatus of tomatoes. Fiziologiya Rastenii, 17: 580-584.

Lazof, D. \& Bernstein, N. (1998). The NaCl-induced inhibition of shoot growth: the case for disturbed nutrition with special consideration of calcium nutrition. Advances in Botanical Research, 29: 113-189.

Levitt, J. (1980). Responses of Plants to Environmental Stresses. 2nd edn. Academic Press, New York.

Maggio, A., Miyazaki, S., Veronese, P., Fujita, T., Ibeas, J. I., Damsz, B., Narasimhan, M. L., Hasegawa, P. M., Joly, R. J. \& Bressan, R. A. (2002). Does proline accumulation play an active role in stress-induced growth reduction. Plant J., 31: 699-712.

Mondal, M. R. I., Islam, M. S., Jalil, M. A. B., Rahman, M. M., Alam, M. S. \& Rahman, M. H. H. (2011). KRISHI PROJUKTI HATBOI (Handbook of Agro-technology), $5^{\text {th }}$ edition. Bangladesh Agricultural Research Institute, Gazipur-1701, Bangladesh, pp: 390-394.

Olympios, C. M., Karapanos, I. C., Lionoudakis, K. \& Apidianakis. I. (2003). The growth, yield and quality of greenhouse tomato in relation to salinity applied at different stages of plant growth. Acta Hort., 609: 313-320.

Oztekin, G. B. \& Tuzel, Y. (2011). Comparative salinity responses among tomato genotypes. Pakistan J. Botany, 43(6): 2665-2672.

Peralta, E., Knapp, S. \& Spooner, O. M. (2005). New species of wild tomato (Solanum section Lycoperisicon: Solanaceae) flom; Iorthem Pem. Systematic Bot., 30: 424-434.

Rahman, M. M. \& Ahsan, M. (2001). Salinity Constrains and Agricultural Productivity in Coastal Saline area of Bangladesh, Journal of Soil Resources in Bangladesh: Assessment and utilization, pp. 1-14.

Rao, G. G. \& Rao, G. R. (1981). Pigment composition \& chlorophyllase activity in pigeon pea (Cajanus indicus Spreng) \& Gingelley (Sesamum indicum L.) under $\mathrm{NaCl}$ salinity. Indian J. of Expet. Biology, 19: 768-770.

Sattar, S., Hussnain, T. \& Javaid, A. (2010). Effect of $\mathrm{NaCl}$ salinity on cotton (Gossypium arboretum L.) grown on MS medium and in hydroponic cultures. The J. Anim. Plant Sci., 20(2): 87-89.

Singh, A. K. \& Dubey, R. S. (1995). Changes in chlorophyll $a$ and $b$ contents and activities of photosystems 1 and 2 in rice seedlings induced by NaCl. Photosynthetica, 31: 489-499.

Sonneveld, C. \& Van der Burg, A. M. M. (1991). Sodium chloride salinity in fruit vegetable crops in soilless culture. Netherlands J. Agri. Sci., 39: 115-122.

SRDI. (2010). Soil Salinity Report 2010 of Bangladesh, Soil Resources Development Institute, Dhaka, Bangladesh.

Tavakkoli, E., Fatehi, F., Coventry, S., Rengasamy, P. \& McDonald, G. (2011). Additive effects of $\mathrm{Na}^{+}$and $\mathrm{Cl}^{-}$ions on barley growth under salinity stress. J. Exp. Bot., 62: 2189-2203.

Vinten, A., Shalhavet, J., Meiri, A. \& Peretz, J. (1986). Water and leaching requirements of industrial tomatoes irrigated with brackish water. Irr. Sci., 7: 13-25.

Werner, J. E. \& Finkelstein, R. R. (1995). Arabidopsis mutants with reduced response to $\mathrm{NaCl}$ and osmotic stress. Physiology of Plant, 93: 659-666. 
Yasar, F., Uzal, O., Tufenkci, S. \& Yildiz, K. (2006). Ion accumulation in different organs of green bean genotypes grown under salt stress. Plant Soil Environ., 52: 476-480.

\section{Citation for this article (APA Style):}

Shiam, I. H., Nahiyan, A. S. M., Momena, K., Mehraj, H. \& Jamal Uddin, A. F. M. (2015). Effect of $\mathrm{NaCl}$ Salt on Vegetative Growth and Yield of Sixteen Tomato Lines. Journal of Bioscience and Agriculture Research 03(01), 15-27. 